\title{
Study on the Mechanism of Acori Graminei Rhizoma in the Treatment of Alzheimer's Disease Based on Network Pharmacology and Molecular Docking
}

\author{
Yi Kuan Du $\left(\mathbb{D},{ }^{1}\right.$ Yue Xiao $\mathbb{D}^{2},{ }^{2}$ Shao Min Zhong $\mathbb{D}^{2}$, Yi Xing Huang $\mathbb{D}^{2}$ Qian Wen Chen $\mathbb{D}^{2}$ \\ Yu Qi Zhou $\mathbb{D}^{2}$, Jin Yan Guo $\mathbb{D}^{2}$, and Chun Yang $\mathbb{D}^{2}$ \\ ${ }^{1}$ Central Laboratory, Affiliated Dongguan Hospital, Southern Medical University, Dongguan, Guangdong, China 523059 \\ ${ }^{2}$ Key Laboratory of Stem Cell and Regenerative Tissue Engineering, Guangdong Medical University Dongguan, China 523808 \\ Correspondence should be addressed to Jin Yan Guo; guojinyan1001@163.com and Chun Yang; yangchunangel@163.com
}

Received 25 April 2021; Accepted 20 October 2021; Published 24 December 2021

Academic Editor: Kazim Husain

Copyright (C) 2021 Yi Kuan Du et al. This is an open access article distributed under the Creative Commons Attribution License, which permits unrestricted use, distribution, and reproduction in any medium, provided the original work is properly cited.

\begin{abstract}
Alzheimer's disease is a common neurodegenerative disease in the elderly. This study explored the curative effect and possible mechanism of Acori graminei rhizoma on Alzheimer's disease. In this paper, 8 active components of Acori graminei rhizoma were collected by consulting literature and using the TCMSP database, and 272 targets were screened using the PubChem and Swiss Target Prediction databases. Introduce it into the software of Cytoscape 3.7.2 and establish the graph of "drug-active ingredient-ingredient target." A total of 276 AD targets were obtained from OMIM, Gene Cards, and DisGeNET databases. Import the intersection targets of drugs and diseases into STRING database for enrichment analysis, and build PPI network in the Cytoscape 3.7.2 software, whose core targets involve APP, AMPK, NOS3, etc. GO analysis and KEGG analysis showed that there were $195 \mathrm{GO}$ items and 30 AD-related pathways, including Alzheimer's disease pathway, serotonin synapse, estrogen signaling pathway, dopaminergic synapse, and PI3K-Akt signaling pathway. Finally, molecular docking was carried out to verify the binding ability between Acori graminei rhizoma and core genes. Our results predict that Acori graminei rhizoma can treat $\mathrm{AD}$ mainly by mediating Alzheimer's signal pathway, thus reducing the production of $\mathrm{A} \beta$, inhibiting the hyperphosphorylation of tau protein, regulating neurotrophic factors, and regulating the activity of kinase to change the function of the receptor.
\end{abstract}

\section{Introduction}

Alzheimer's disease $(\mathrm{AD})$ is a neurodegenerative disease characterized by a severe decline in cognitive function and memory. The main pathological features were abnormal deposition of $\mathrm{A} \beta$ and nerve fiber tangles formed by hyperphosphorylated tau protein accumulation $[1,2]$. At present, cholinesterase inhibitors are commonly used to improve the patients' cognitive function with $\mathrm{AD}$ [3], but these drugs can only alleviate the disease but cannot reverse the disease's progression. Further studies have shown that the etiology of Alzheimer's disease may be related to a series of complex factors, such as heredity and obesity [4]. In diseases with complex pathogenesis, the study of the "multi-component, multi-target" drug action mechanism plays a prominent role [5]. As a multicomponent and multitarget discipline system, network pharmacology has its unique advantages in studying complex molecular mechanisms $[6,7]$.

In traditional Chinese medicine, $\mathrm{AD}$ is often classified as "dementia" caused by brain marrow deficiency and evil, disturbing brain orifices [8]. The data show that traditional Chinese medicine has used buxu, kaiqiao, dissipation blood stasis, and activating blood circulation to treat $\mathrm{AD}$, and the curative effect is reliable $[9,10]$. Acori graminei rhizoma (AGR) is a famous Chinese herbal medicine with a pungent taste and warm nature. It has the effect of Xingshen Yizhi and occupies the first place in the single traditional Chinese medicine for the treat $\mathrm{AD}$. Acori graminei rhizoma is widely used in many fields, such as Alzheimer's disease, epilepsy, amnesia, aphasia caused by stroke, and tinnitus. Furthermore, it has been found that Acori graminei rhizoma can play the role of anti- $\beta$-amyloid protein $(\mathrm{A} \beta)$ deposition, 
TABLE 1: Information of 8 active chemical constituents selected from Acori graminei rhizoma.

\begin{tabular}{lccccc}
\hline Molecule ID & Molecule name & Component number & OB & DL & BBB \\
\hline MOL002955 & $2^{\prime}$-O-Methylisoliquiritigenin & AGR1 & 75.86 & 0.62 & -0.16 \\
MOL003571 & Spathulenol & AGR2 & 81.61 & 0.78 \\
MOL003553 & Calamendiol & AGR3 & 61.13 & 0.18 \\
MOL000269 & Elemicin & AGR4 & 21.94 & 0.06 \\
MOL003549 & Gamma-asarone & AGR5 & 22.76 & 0.06 \\
MOL002124 & $\beta$-Asarone & AGR6 & 35.61 & 1.55 \\
MOL003547 & $\alpha$-Asarone & AGR7 & 38.39 & 0.06 \\
MOL000254 & Eugenol & AGR8 & 56.24 & 0.06 & 0.04 \\
\hline
\end{tabular}
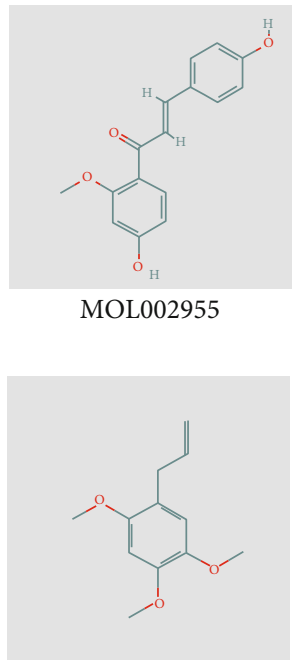

MOL003549

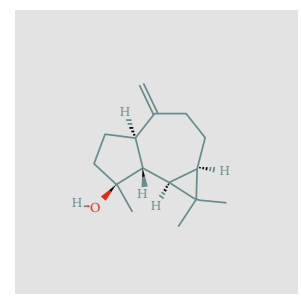

MOL003571

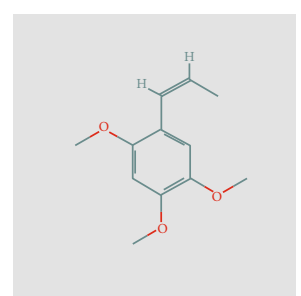

MOL002124

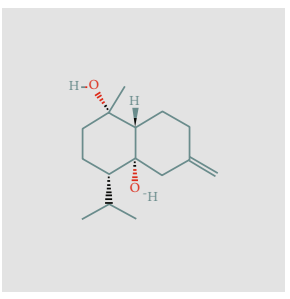

MOL003553

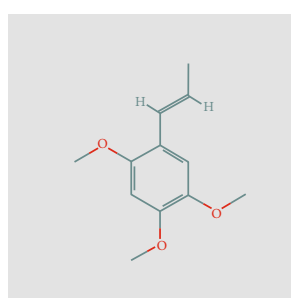

MOL003547

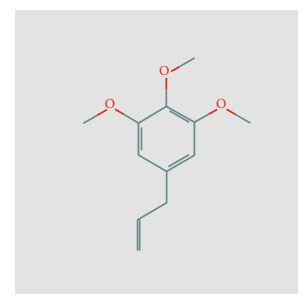

MOL000269

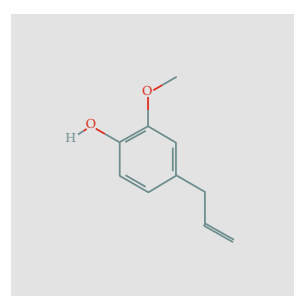

MOL000254

Figure 1: Structure of 8 active components selected from Acori graminei rhizoma.

anticentral cholinergic nerve function damage, and antineuronal apoptosis $[11,12]$. However, the pharmacological research on the treatment of $\mathrm{AD}$ is not perfect. In this paper, the network relationship between active components and targets of Acori graminei rhizoma and AD was analyzed by using the method of network pharmacology to provide a molecular study on the mechanism of action of Acori graminei rhizoma in treating $\mathrm{AD}$.

\section{Materials and Methods}

2.1. Screening of Components of Acori Graminei Rhizoma. Acori graminei rhizoma's chemical constituents were obtained by consulting the literature and TCMSP database (http://lsp.nwu.edu.cn/tcmsp.php). In this study, the chemical components of Acori graminei rhizoma were screened based on the pharmacokinetic parameters: absorption, distribution, metabolism, and excretion (ADME). The screening conditions were oral bioavailability $(\mathrm{OB}) \geq 30 \%$ and drug - like $(D L) \geq 0.18$. Additionally, a chemical acting on a specific part of the brain must effectively penetrate the blood-brain barrier (BBB) to reach the brain target. To study the mechanism of action of Acori graminei rhizoma on nervous system diseases, BBB can be used as a screening index, and the threshold value of $\mathrm{BBB} \geq-0.30$ (it is considered that the molecule has certain permeability) is used as a threshold. Finally, the active components of Acori graminei rhizoma, which are not included, are collected and supplemented by literature.

2.2. Acquisition of Component Targets. Import the components into the PubChem database (https://Pubchem.ncbi .nlm.nih.gov/), search and collect the 2D structure of the components, import them into the Swiss Target Prediction database (http://www.swisstargetprediction.ch/), and obtain the component targets. Then, using the target protein name in the Uniprot database (http://www.Uniprot.org), the genes were standardized and sorted out, and finally, possible target genes for the compound's action were downloaded and sorted out.

2.3. Acquisition of $A D$ Targets. Search for "Alzheimer's disease" in OMIM database (https://www.omim.org/), CTD database (http://CTD.mdibl.org/), and Gene Cards database (https://www.genecards.org/). Using Excel combined data, the target protein was introduced into the Uniprot database for gene standardization, and coincident genes were removed to collect $\mathrm{AD}$ disease genes. 


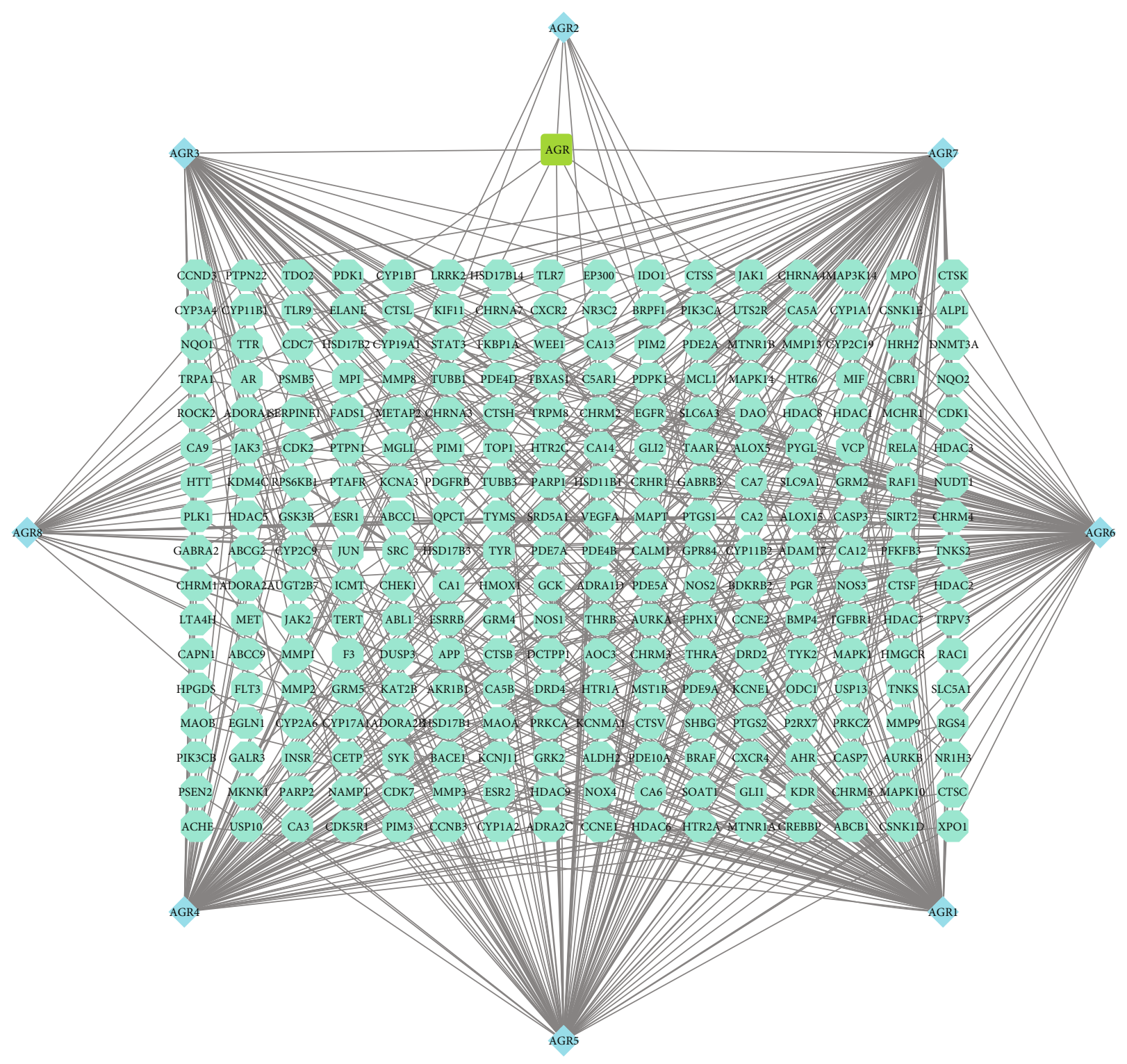

Figure 2: "Drug-active ingredient-component target" network.

2.4. Construction of "Drug-Active Ingredient-Target" Network. The molecular formulas of the Acori graminei rhizoma components were expressed by AGR1-AGRn (AGR is the abbreviation of "Acori graminei rhizoma"). The components and corresponding targets were introduced into the Cytoscape3.7.2 software to construct the "drug-active ingredient-target" network and then analyze it. In the network, the active components and protein targets are represented by a node, and an edge connects the nodes. Acori graminei rhizoma's core targets in $\mathrm{AD}$ treatment were analyzed according to the betweenness centrality (BC), closeness centrality (CC), and the median of Degree.

2.5. Construction of Intersection Target Network between Drugs and Diseases. The intersection gene was introduced into Wei Shengxin website (http://bioinfogp.cnb.csic.es/ tools/Venn/index.html), and the Venn diagram was used to show the common target of AGR and AD. The intersec- tion gene was introduced into the STRING database (https://string-db.org/) and searched under the condition of Homo sapiens. 0.40 and 0.70 were used as the moderate correlation threshold and high correlation threshold of Acori graminei rhizoma. The Node1 and Node2 in the results were introduced into the Cytoscape 3.7.2 software. The topological analysis and calculation were carried out using the Network Analyzer tool to construct the "componenttarget-disease" network. The core target was predicted according to the median of BC, CC, and Degree value.

2.6. GO Analysis and KEGG Analysis. Gene ontology (GO) analysis and Kyoto Encyclopedia of Gene and Genome (KEGG) analysis can provide gene expression data and systematic visualization information of target genes. In this study, GO analysis and KEGG functional annotation and enrichment analysis were carried out by using DAVID 6.8 database (https://david.ncifcrf.gov/). According to " $P \leq 0.01$ " 


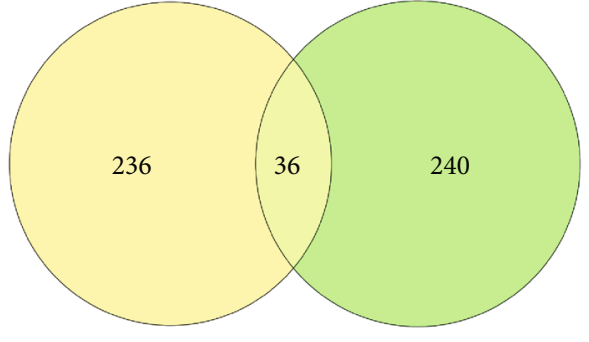

Acori graminei rhizoma Alzheimer's disease

Figure 3: Venn diagram of the overlapping genes of Acori graminei rhizoma and Alzheimer's disease.

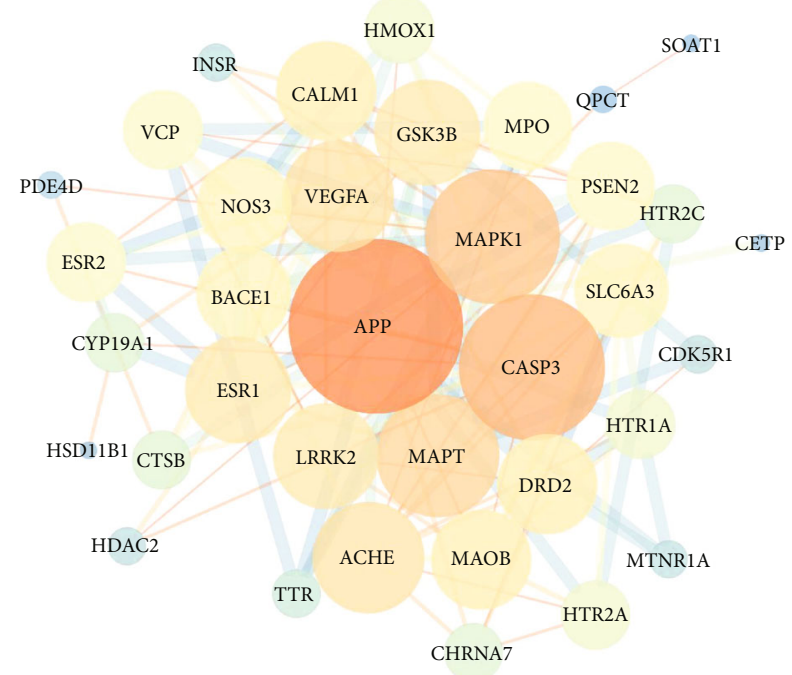

FIgURE 4: Construction of protein-protein interaction network of Acori graminei rhizoma under 0.4 threshold. 36 nodes represent 36 proteins, and 138 edges represent the interactions between 138 pairs of proteins. The size and color of the node represent the degree, and the size and color of the edge represent the comprehensive score.

on the Wei Shengxin website (http://www.bioinformatics .com.cn/), significantly enriched biological annotations were screened out according to biological information. GO analysis chart and KEGG analysis bubble chart were drawn to predict the pathway of Acori graminei rhizoma in $\mathrm{AD}$ treatment.

2.7. Molecular Docking of "Drug Components-Core Targets." The LEDOCK molecular docking software was used to verify the molecular docking, between Acori graminei rhizoma's active components and the core targets in the network analysis, to explore the binding degree between the active components and the core targets.

\section{Results}

3.1. Screening of Active Chemical Constituents of Acori Graminei Rhizoma. In this study, there were a total of 105
TABLE 2: Topological values of core targets in protein-protein interaction networks.

\begin{tabular}{lccc}
\hline Core target & Betweenness centrality & Closeness centrality & Degree \\
\hline APP & 0.36432506 & 0.72916667 & 23 \\
CASP3 & 0.12727436 & 0.66037736 & 18 \\
MAPK1 & 0.10814869 & 0.625 & 16 \\
MAPT & 0.05142191 & 0.60344828 & 14 \\
VEGFA & 0.05260249 & 0.58333333 & 12 \\
ACHE & 0.03699421 & 0.56451613 & 12 \\
GSK3B & 0.02680704 & 0.57377049 & 11 \\
ESR1 & 0.04004646 & 0.58333333 & 11 \\
LRRK2 & 0.01789274 & 0.56451613 & 11 \\
DRD2 & 0.03274326 & 0.53030303 & 10 \\
\hline
\end{tabular}

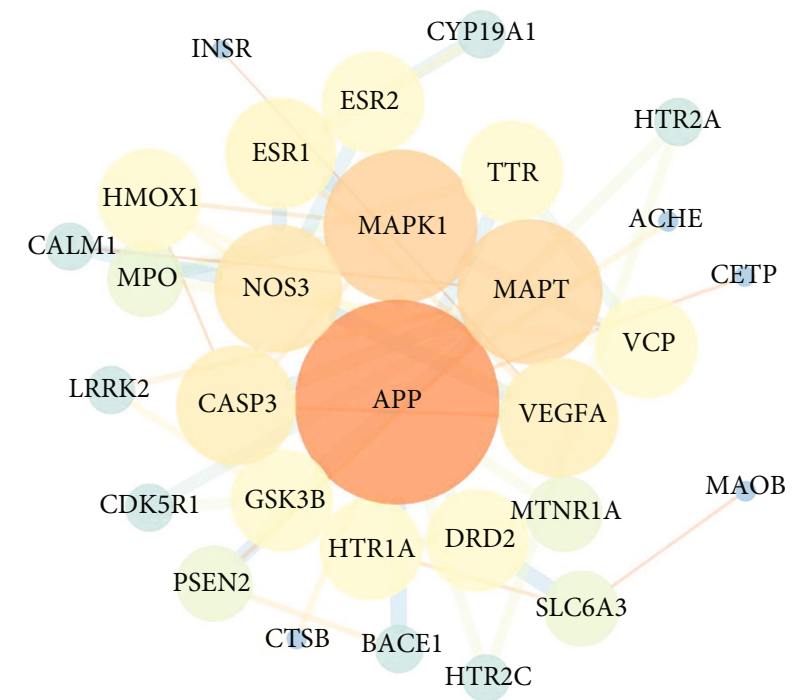

FIGURE 5: Construction of a protein-protein interaction network expressed by a common target at a threshold of 0.7. 30 nodes represent 30 proteins, 59 edges represent 59 pairs of protein interactions, node size and color represent, and edge size and color represent comprehensive scores.

chemical constituents of Acori graminei rhizoma in the TCMSP database. Three components were screened and numbered according to the conditions of $\mathrm{OB} \geq 30 \%, \mathrm{DL} \geq$ 0.18 , and $\mathrm{BBB} \geq-0.30$, including $2^{\prime}$-O-methylisoliquiritigenin, calamendiol, and spathulenol. The molecule ID in the table is the identity information of the component, the name of molecule is the compound name of the ingredient, and the component number represents the coding sorted by the acronym of Acori graminei rhizoma.

Modern studies have shown that elemicin, gammaasarone, $\beta$-asarone, $\alpha$-asarone, and eugenol are significant constituents in Acori graminei rhizoma, while ADME parameters of the above ingredients are outside the range of screening in the TCMSP database. Our group added them to the list, so there are eight components in total, as shown in Table 1 and Figure 1. 


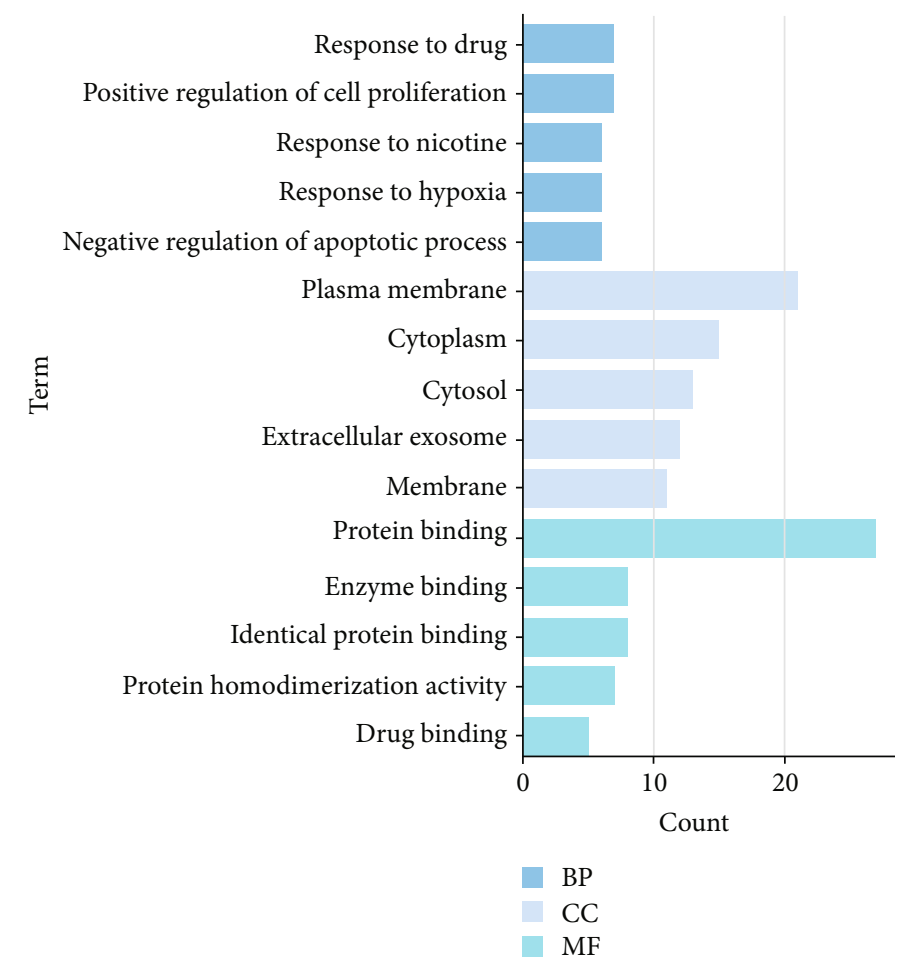

FIGURE 6: GO analysis results. The results of biological processes show that Acori graminei rhizoma mainly mediates the response of the disease to drugs and participates in signal transduction. The results of cell composition show that the plasma membrane and cytoplasm are the key regions. The results of molecular function study show that binding to protein is the main pathway of action.

3.2. Targets of Drug Ingredients. Imported the above eight components into the PubChem database and Swiss Target Prediction database and obtained the effective component targets of Acori graminei rhizoma. The result included 657 targets, and after removing the repetition value, there remained 272 targets in total.

3.3. Targets for AD Disease. Entered “Alzheimer's disease” into the OMIM database, Gene Cards database, and CTD database to get the disease target, where the relevance score or inference is greater than 0. Finally, we combined the database information and deleted the coincident genes. Then, the result consisted of 276 pieces of target information related to $\mathrm{AD}$ diseases.

3.4. Construction and Analysis of the Drug-Target Network. We used the Cytoscape 3.7.2 software to construct a PPI network of drug-active ingredient-component targets based on the previous results. The blue diamond represents the active compound of Acori graminei rhizoma, the cyan octagonal represents the target gene, and the green square represents Acori graminei rhizoma, as shown in Figure 2. The network has 281 nodes ( 1 drug node, eight active component nodes, 272 target nodes, and 577 edges). Network Analyzer calculates the PPI network. The median of BC and CC was 0.00006847 and 0.318182 , respectively, and the median of 2 times of Degree value was 4 . The core node needs to meet the median card value of the above parameters. The value of $\beta$-asarone and $\alpha$-asarone is higher, followed by $2^{\prime}-\mathrm{O}$-methylisoliquiritigenin and other components. These active com- ponents with higher parameters may play a relatively important role in treating $\mathrm{AD}$ disease, and these components may be drug docking targets.

3.5. Construction and Analysis of "Drug Ingredient-TargetDisease" Network. The Venn diagram of the intersection target was drawn through the Wei Shengxin website to analyze the intersection target between the component target of Acori graminei rhizoma and AD. As shown in Figure 3, we found 36 intersection targets between the component target of Acori graminei rhizoma and $\mathrm{AD}$, and the common targets were APP, CASP3, MAPK1, ACHE, and others.

The purpose of uploading the above 36 targets to the STRING database was to draw the protein-protein interaction (PPI) network between drugs and diseases and then gained the visual analysis through the Cytoscape 3.7.2 software. The size and color of the node are correlated with the Degree value positively. The larger the Degree value, the larger the node and the darker the color, indicating that the target is more important in this network relationship. A PPI network with a threshold of 0.4 is constructed (Figure 4). BC $\geq 0.00819305, \mathrm{CC} \geq 0.493055555$, and Degree $\geq 14$ (2 times median) are calculated by the Cytoscape 3.7.2 software. The core targets APP, CASP3, MAPK1, MAPT, VEGFA, ACHE, GSK3B, ESR1, LRRK2, DRD2, and so on, which are located in the center of the network and have a high overall score, are selected and used as molecular docking targets (Table 2). To calculate $\mathrm{BC} \geq 0.00400246$, $C C \geq 0.4084507$, and Degree $\geq 6$ ( 2 times the median), we constructed a PPI network with a threshold of 0.7 


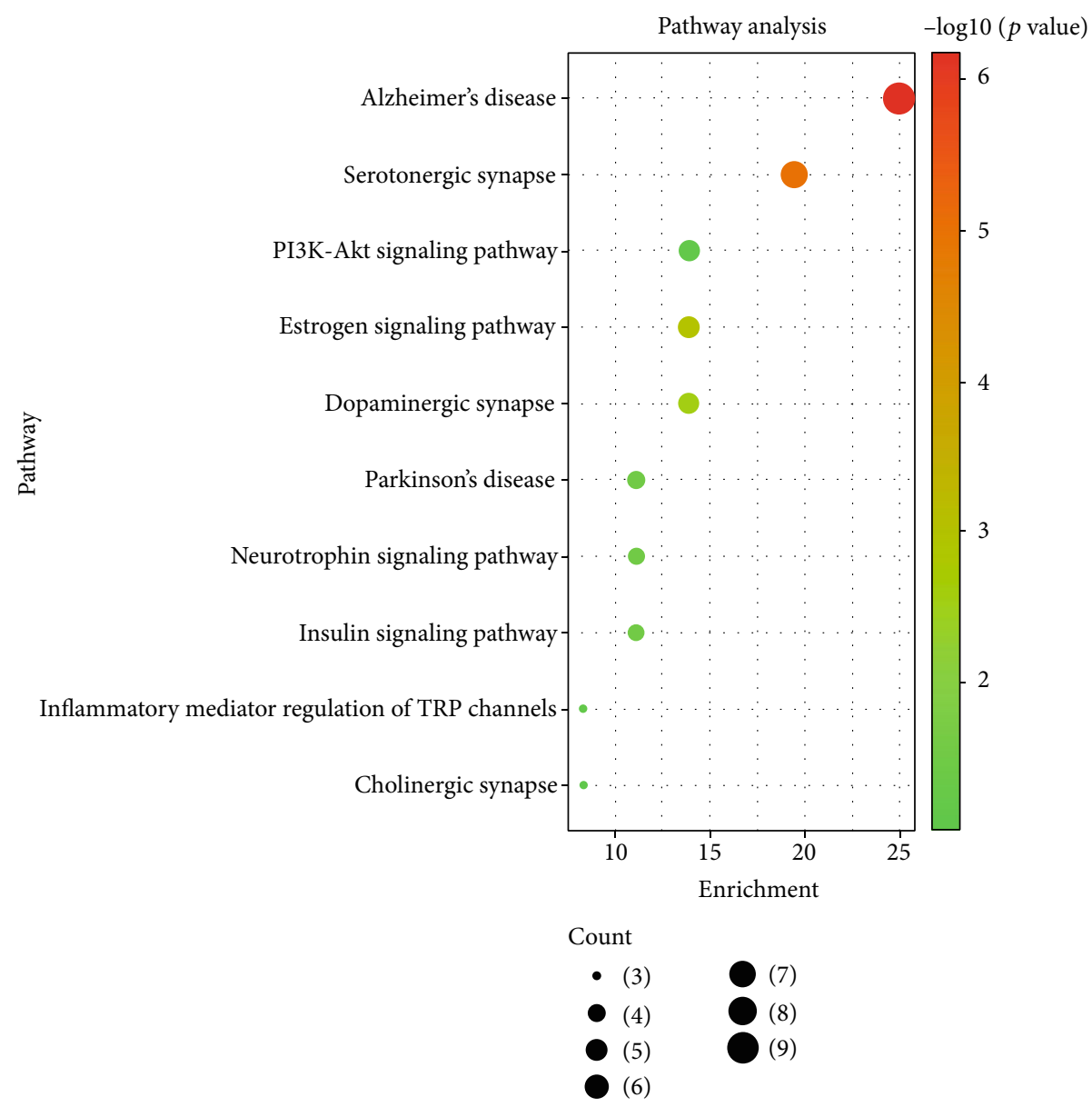

FIGURE 7: KEGG results show that related pathways include Alzheimer's disease, serotonergic synapses, estrogen signal pathways, dopaminergic synapses, etc.

(Figure 5), calculated the common target genes, and deleted the unconnected nodes in a further study. The network shows that APP, MAPK1, MAPT, NOS3, VEGFA, and CASP3 are situated in the network center. The results of the central target of Acori graminei rhizoma in the treatment of $\mathrm{AD}$ disease suggest that it may play an efficient role in the pharmacological action of Acori graminei rhizoma.

3.6. Functional Pathway Annotation of Drug Ingredient Targets. In this study, a total of 195 GO items were enriched, and the first $15 \mathrm{GO}$ items with the lowest $P$ value were selected for mapping $(P<0.05)$, as shown in Figure 6 . The $Y$-axis represents the GO entry, and the area size of the $X$-axis and bar chart represents the number of genes belonging to GO in the target gene set. In the biological process (BP), the typical targets are mainly concentrated in the response to drugs, the positive regulation of cell proliferation, the response to nicotine, the response to hypoxia, the negative regulation of apoptosis, and so on. In the cellular component (CC), the typical targets are mainly concentrated in the plasma membrane, cytoplasm, extracellular body, membrane, etc. In terms of molecular functional (MF), the typical targets are mainly related to protein binding, enzyme bind- ing, same protein binding, protein homodimerization activity, drug binding, and so on.

We obtained a total of 30 enrichment pathways through KEGG pathway enrichment and drew the bubble diagram according to the first ten pathways with the lowest $P$ value combined with biological annotations (Figure 7). $Y$-axis represents the name of the pathway, $X$-axis and bubble area represent the number of genes belonging to this signal pathway in the target gene set, and bubble color represents enrichment significance, that is, the size of $P$ value. These common targets are enriched in Alzheimer's disease, serotonergic synapses, HIF-1 signal pathways, estrogen signaling pathways, alcoholism, cocaine addiction, dopaminergic synapses, prolactin signal pathways, gap junctions, and neuroactive ligand-receptor interactions. The analysis of this series of practical tests can provide valuable information to explain the possible mechanism of Acori graminei rhizoma in $\mathrm{AD}$ treatment.

3.7. Results of "Drug Component-Core Target" Molecule Docking. The study used ten core targets with a high score in protein-protein interaction for molecular docking. Since there was no data on LRRK2, the rest nine core targets were selected to conduct molecular docking with Acori graminei 
TABLE 3: Results of molecular docking of key targets and their corresponding compounds.

\begin{tabular}{|c|c|c|c|}
\hline Serial number & Core target & Compound & LeDock score \\
\hline 1 & & $\alpha$-Asarone & $-11.83 \mathrm{~kJ} / \mathrm{mol}$ \\
\hline 2 & APP & $\beta$-Asarone & $-11.58 \mathrm{~kJ} / \mathrm{mol}$ \\
\hline 3 & & $2^{\prime}$-O-Methylisoliquiritigenin & $15.72 \mathrm{~kJ} / \mathrm{mol}$ \\
\hline 4 & & $\alpha$-Asarone & $-14.00 \mathrm{~kJ} / \mathrm{mol}$ \\
\hline 5 & CASP3 & $\beta$-Asarone & $-14.04 \mathrm{~kJ} / \mathrm{mol}$ \\
\hline 6 & & $2^{\prime}$-O-Methylisoliquiritigenin & $-24.45 \mathrm{~kJ} / \mathrm{mol}$ \\
\hline 7 & & $\alpha$-Asarone & $-14.63 \mathrm{~kJ} / \mathrm{mol}$ \\
\hline 8 & MAPK1 & $\beta$-Asarone & $-15.05 \mathrm{~kJ} / \mathrm{mol}$ \\
\hline 9 & & $2^{\prime}$-O-Methylisoliquiritigenin & $-23.12 \mathrm{~kJ} / \mathrm{mol}$ \\
\hline 10 & & $\alpha$-Asarone & $-14.55 \mathrm{~kJ} / \mathrm{mol}$ \\
\hline 11 & MAPT & $\beta$-Asarone & $-14.63 \mathrm{~kJ} / \mathrm{mol}$ \\
\hline 12 & & $2^{\prime}$-O-Methylisoliquiritigenin & $-23.24 \mathrm{~kJ} / \mathrm{mol}$ \\
\hline 13 & & $\alpha$-Asarone & $-14.80 \mathrm{~kJ} / \mathrm{mol}$ \\
\hline 14 & VEGFA & $\beta$-Asarone & $-14.80 \mathrm{~kJ} / \mathrm{mol}$ \\
\hline 15 & & $2^{\prime}$-O-Methylisoliquiritigenin & $-19.31 \mathrm{~kJ} / \mathrm{mol}$ \\
\hline 16 & & $\alpha$-Asarone & $-15.51 \mathrm{~kJ} / \mathrm{mol}$ \\
\hline 17 & ACHE & $\beta$-Asarone & $-15.59 \mathrm{~kJ} / \mathrm{mol}$ \\
\hline 18 & & $2^{\prime}$-O-Methylisoliquiritigenin & $-24.70 \mathrm{~kJ} / \mathrm{mol}$ \\
\hline 19 & & $\alpha$-Asarone & $-13.54 \mathrm{~kJ} / \mathrm{mol}$ \\
\hline 20 & GSK3B & $\beta$-Asarone & $-13.60 \mathrm{~kJ} / \mathrm{mol}$ \\
\hline 21 & & $2^{\prime}$-O-Methylisoliquiritigenin & $-22.74 \mathrm{~kJ} / \mathrm{mol}$ \\
\hline 22 & & $\alpha$-Asarone & $-15.35 \mathrm{~kJ} / \mathrm{mol}$ \\
\hline 23 & ESR1 & $\beta$-Asarone & $-15.38 \mathrm{~kJ} / \mathrm{mol}$ \\
\hline 24 & & $2^{\prime}$-O-Methylisoliquiritigenin & $-23.49 \mathrm{~kJ} / \mathrm{mol}$ \\
\hline 25 & & $\alpha$-Asarone & $-14.84 \mathrm{~kJ} / \mathrm{mol}$ \\
\hline 26 & DRD2 & $\beta$-Asarone & $-15.17 \mathrm{~kJ} / \mathrm{mol}$ \\
\hline 27 & & $2^{\prime}$-O-Methylisoliquiritigenin & $-21.57 \mathrm{~kJ} / \mathrm{mol}$ \\
\hline
\end{tabular}

rhizoma. The docking score is shown in Table 3. It is generally believed that a score of less than $5 \mathrm{~kJ} / \mathrm{mol}$ indicates a good binding activity between the compound and the target. The molecular docking results show that the score of core compounds ( $\beta$-asarone, $\alpha$-asarone, $2^{\prime}$-O-methylisoliquiritigenin, gamma-asarone) and core targets (APP, CASP3, MAPK1, ACHE) is less than $5 \mathrm{~kJ} \mathrm{~mol}^{-1}$, indicating that the core compounds have good binding activity with core targets. The docking mode between $\beta$-asarone and nine core targets is shown in Figure 8.

\section{Discussion}

Studies have shown that among the single drugs of traditional Chinese medicine in treating Alzheimer's disease, Acori graminei rhizoma appears with the highest frequency $[13,14]$, so we choose Acori graminei rhizoma as the research object, which has a significant clinical application value. Eight candidate active components such as $\alpha$-asarone, $\beta$-asarone, and eugenol in Acori graminei rhizoma were screened in this study. The "drug-active ingredient-target" network diagram was constructed by the Cytoscape 3.7.2 software, and it was found that a complex interaction between components and target. The PPI network of "component-target-disease" is constructed based on the STRING database. According to the topology parameters, the factors with high scores are APP, CASP3, MAPK1, MAPT, VEGFA, ACHE, GSK3B, ESR1, LRRK2, DRD2, and so on. A close relationship exists between amyloid precursor protein (APP) and $\beta$-amyloid protein (A $\beta$ ) accumulation. APP produces A $\beta$ through secretase cleavage. Excessive secretion and accumulation of $\mathrm{A} \beta$ in the brain will cause cytotoxicity and injury, cause an inflammatory reaction, and finally lead to nerve cell apoptosis and degenerative lesions [15-17]. Caspase-3, as a highly enriched molecule in PPI analysis, can directly and effectively cleave APP to the direction of $\mathrm{A} \beta$ formation [18]. Interestingly, acetylcholinesterase (AChE) can not only hydrolyze acetylcholine (ACh) but also accelerate $\mathrm{A} \beta$ aggregation and amyloid fibril formation [19]. The D2 receptor in the dopamine-related pathway is closely related to the abnormal accumulation of tau protein. Clinical use of D2 receptor antagonists can reduce the concentration of insoluble tau protein and neurotoxicity [20]. The change of glycogen synthesis kinase (GSK3 $\beta$ ) activity affects tau hyperphosphorylation and participates in AD's formation and development [21]. The activity of 

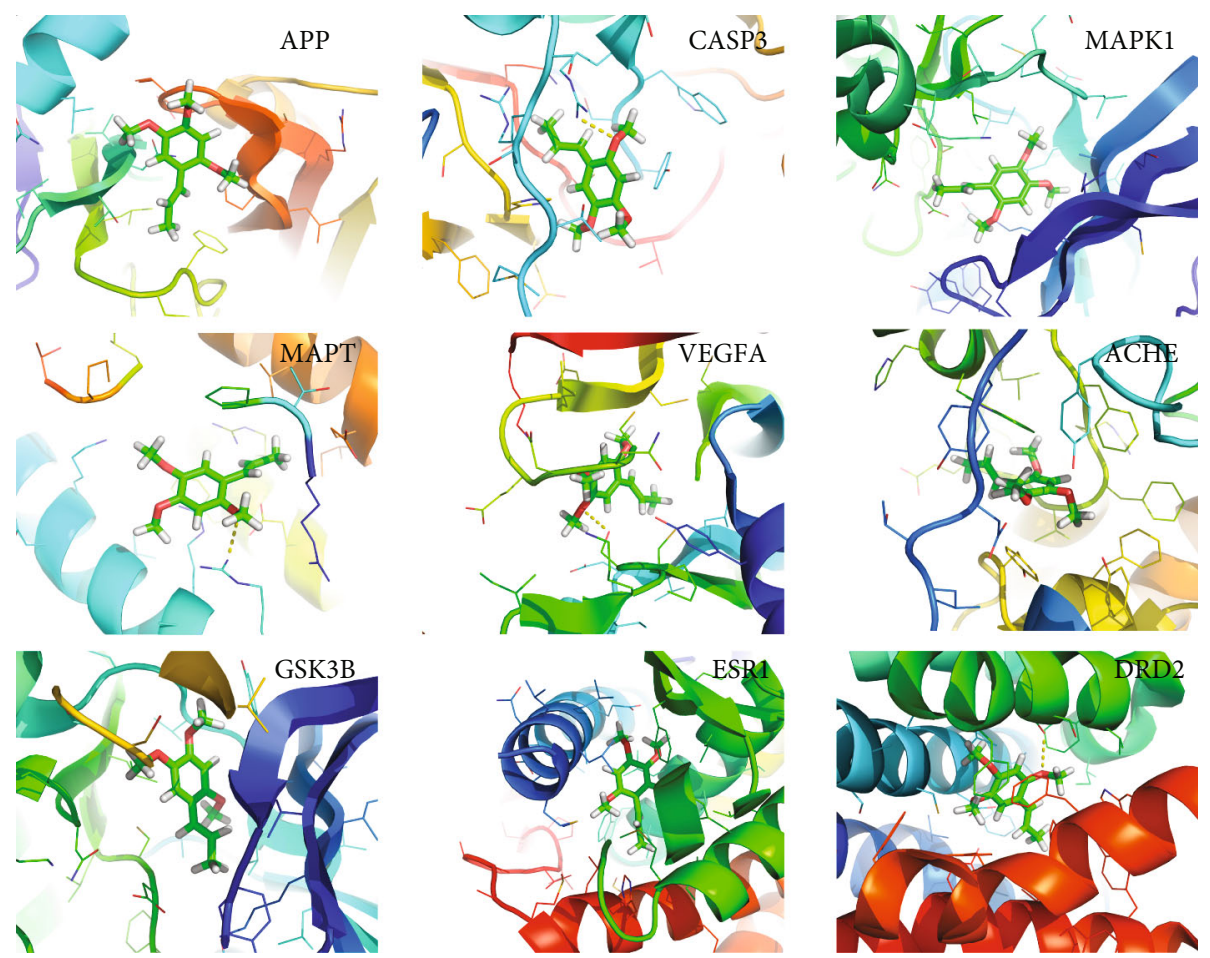

Figure 8: Molecular docking diagram of $\beta$-asarone and core target.

GSK3 $\beta$ is regulated by the classical antiapoptotic signal pathway phosphatidylinositol 3-kinase/protein kinase B (PI3K/AKT) pathway [22-24]. At the same time, mitogenactivated protein kinase (MAPKs) is another important pathway to regulate cell growth and apoptosis, in which the p38MAPK pathway is also related to the pathogenesis of $\mathrm{AD}$. The activation of $\mathrm{p} 38 \mathrm{MAPK}$ leads to hyperphosphorylation of tau, which leads to neurofibril entanglement. p38MAPK accelerates the course of AD by producing neurotoxic proinflammatory factors, increasing $A \beta$ deposition, and activating caspase- 3 to induce apoptosis of hippocampal neurons [25].

In this study, 10 key targets of Acori graminei rhizoma in treating $\mathrm{AD}$ were obtained, but the LRRK2 lacked relevant data so that the rest nine targets were selected for molecular docking with drugs. The molecular docking of $2^{\prime}$-O-methylisoglycine to the core target is very strong, which suggests that it has a research prospect. In molecular docking, we found that $\beta$-asarone has a strong binding activity with APP, and it also has a good performance in the docking diagram. In the process of consulting the relevant literature, we found that $\beta$-asarone can indeed affect the related targets of $\mathrm{AD}$, which suggests that $\beta$-asarone may be a key component in treating $\mathrm{AD}$. $\beta$-asarone may reduce the production of $\mathrm{A} \beta$ and reduce the toxic damage of $\mathrm{A} \beta$ to neurons and synaptic ultrastructure by inhibiting the overexpression of APP or promoting the decomposition and excretion of APP [26]. At the same time, $\beta$-asarone can inhibit the phosphorylation of JNK in hippocampal neurons, upregulate the expression of Bcl-2 protein, and downregulate the expression of caspase- 3 at the transcriptional level, thus play a role in antiapoptosis of hippocampal neurons [27]. Other studies have shown that $\beta$-asarone in the volatile oil of Acori graminei rhizoma can restore the phosphorylation level of GSK-3 $\beta$ and activate $\mathrm{Wnt} / \beta$-catenin pathway, thus reducing $\mathrm{AD}$ caused by tau hyperphosphorylation and $\mathrm{A} \beta$ accumulation [28]. Furthermore, $\beta$-asarone has the effect of acetylcholine inhibitor to reduce $\mathrm{AChE}$ and can inhibit the production of A $\beta 42$ [29], which can alleviate the AD's development. Interestingly, other components of Acori graminei rhizoma also play a specific role in interfering with $\mathrm{AD}$. Eugenol in the component inhibits NF- $\kappa \mathrm{B}$ and MAPK pathway and has antiapoptosis and antioxidant activities and may also play a role in treating $\mathrm{AD}[30,31] . \alpha$-Asarone can protect glial cells by inhibiting the release of inflammatory cytokines [32, 33].

To explain the selected key targets' role in gene function and signal pathway, GO functional enrichment analysis and KEGG pathway enrichment analysis were carried out in this study. According to the analysis results, some common targets are enriched in Alzheimer's disease, serotonergic synapses, HIF-1 signal pathways, estrogen signal pathways, dopaminergic synapses, neuroactive ligand-receptor interactions, and so on. Serotonin can maintain nerve excitability. When its expression decreased, it will aggravate brain neuron damage and clinical manifestation of cognitive impairment [34]. Eugenol in Acori graminei rhizoma can normalize the concentration of 5-hydroxytryptamine in the brain [35], which helps recover nerve injury and improve $\mathrm{AD}$ symptoms. In the treatment of $\beta$-asarone combined with $\mathrm{L}$-dopa, it was found that $\beta$-asarone may reduce dopaminergic neurons' damage by regulating the HSP70/MEF2D/ Beclin1 pathway in rats [36]. It corresponds to the dopaminergic synapses in KEGG analysis, and the dopamine system plays an efficient role in the pathological process of $\mathrm{AD}$. 


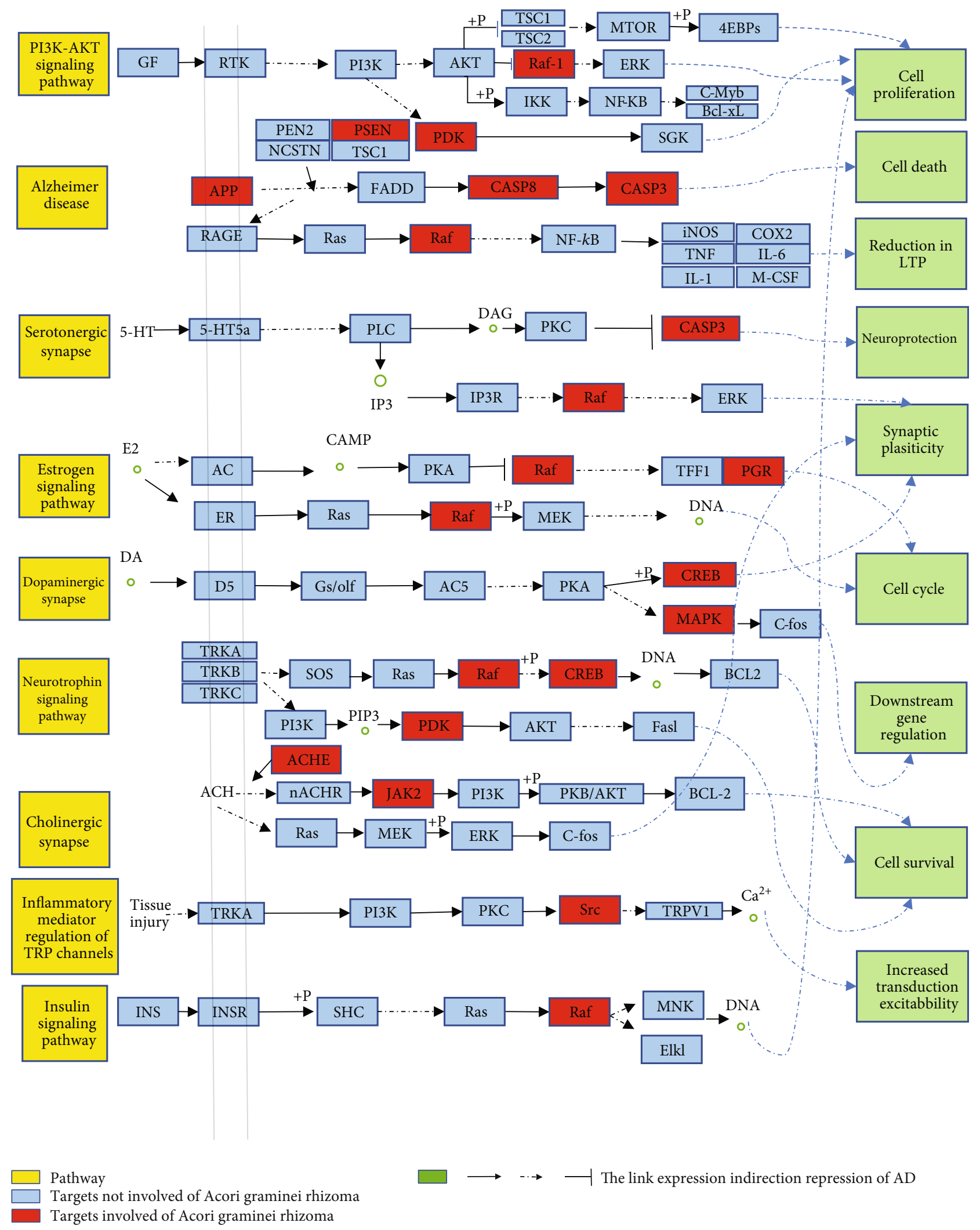

FIgURE 9: Hypothetical mechanism of Acori graminei rhizoma in the treatment of AD (the yellow box is the signal pathway, the red box is the target of the direct action of the drug, and the green box is the participating biological process). 
Interestingly, only moderate dopamine signals can optimize the cognitive function of brain [37]. Estrogen receptor (ESR1) is one of our group's core targets [38], and the estrogen pathway is one of the crucial pathways obtained from enrichment analysis. Studies have shown that estrogen has a neuroprotective effect on $\mathrm{AD}$ [39], but the expression of estrogen or ESR1 in AD is downregulated [40]. $\beta$-Asarone, one of the main components of Acori graminei rhizoma, decreased the concentration of calcium, inhibited apoptosis, and alleviated the dementia damage of vascular endothelial cells induced by $\beta$-amyloid protein in the AD cell model [41]. Studies have shown that ESR1 may participate in AD's pathophysiology by regulating the transport of calcium ions. However, there are no specific studies to show whether Acori graminei rhizoma can regulate calcium concentration through the estrogen pathway to restore normal brain function by affecting ESR1. It needs further exploration. In order to facilitate the discussion and analysis of the mechanism of Acorus tatarinowii in the treatment of Alzheimer's disease, our research team selected the more relevant signal pathways, action targets, and biological processes and made a hypothetical mechanism diagram (Figure 9).

\section{Conclusion}

To sum up, in this study, the network pharmacology method was used to analyze the complex network relationship between multicomponents as well as multitargets of Acori graminei rhizoma and $\mathrm{AD}$ disease targets, which was to explore the molecular mechanism of Acori graminei rhizoma in the treatment of AD. As a result, we found prominent signal molecules such as APP, CASP3, and MAPK1, as well as key signal pathways like Alzheimer's disease and serotonergic synapses. Among them, pathways, for instance, alcoholism and cocaine addiction, are closely related to the pathogenesis, suggesting that these potential pathways are worth studying. At the same time, we predict that the main components of Acori graminei rhizoma in treating of $\mathrm{AD}$ are $\alpha$-asarone and $\beta$-asarone. The therapeutic effect of Acori graminei rhizoma on $\mathrm{AD}$ has been carried out clinically, and the curative effect is remarkable. On this basis, this study preliminarily verified the pharmacological mechanism of Acori graminei rhizoma in the treatment of $\mathrm{AD}$, which laid a foundation for further research from the level of cell biology.

\section{Data Availability}

The data used to support the findings of this study are included within the article.

\section{Conflicts of Interest}

The authors have no conflicts of interest.

\section{Authors' Contributions}

Chun Yang and YiKuan Du contributed to the experimental design and financial support for this study. Yue Xiao, ShaoMin Zhong, YuQi Zhou, and JinYan Guo contributed to carry out the experimental design and data analysis and wrote the paper. QianWen Chen performed the molecule docking analysis. YiXing Huang drew the hypothetical mechanism diagram. All authors read and approved the final manuscript. Yi Kuan Du and Yue Xiao contributed equally to this study.

\section{Acknowledgments}

This work was supported by the Natural Science Foundation of Guangdong Province (No. 2020A1515010011; No. 2021A1515011580), the Medical Scientific Research Foundation of Guangdong Province (No. A2020096; No. B2021330), the Administration of Traditional Chinese Medicine of Guangdong Province (No. 20202101; No. 20211216), the Dongguan Science and Technology of Social Development Program (No. 20211800905342), the Research and Development Fund of Dongguan People's Hospital (No. k201902), the Special Funds for the Cultivation of Guangdong College Students' Scientific and Technological Innovation (Climbing Program Special Funds) (No. pdjh2020a0255; No. pdjh2021b0224), the Guangdong medical university students' Innovation experiment program (No. 2019ZZDS007; No. 2019ZZDS003; No. 2020ZZDS002; No. 2020ZYDS005), the Guangdong medical university students' Innovation and entrepreneurship training program (No. GDMU2019003; Nos. GDMU2019041, GDMU2020010, and GDMU2020078), the Provincial and national college students' innovation and entrepreneurship training program (No. 202010571003; No. S202010571041; No. 202110571010; No. S202110571078), and the Scientific Research Fund of Guangdong Medical University (No. GDMUZ2020009).

\section{References}

[1] H. W. Querfurth and F. M. LaFerla, “Alzheimer's disease,” The New England Journal of Medicine, vol. 362, no. 4, pp. 329-344, 2010.

[2] L. M. Ittner and J. Gotz, "Amyloid- $\beta$ and tau - a toxic pas de deux in Alzheimer's disease," Nature Reviews Neuroscience, vol. 12, no. 2, pp. 67-72, 2011.

[3] S. K. Shrivastava, S. K. Sinha, P. Srivastava et al., "Design and development of novel p-aminobenzoic acid derivatives as potential cholinesterase inhibitors for the treatment of Alzheimer's disease," Bioorganic Chemistry, vol. 82, pp. 211-223, 2019.

[4] J. Mendiola-Precoma, L. C. Berumen, K. Padilla, and G. Garcia-Alcocer, "Therapies for prevention and treatment of Alzheimer's disease,", Biomed Research International, vol. 2016, Article ID 2589276, 17 pages, 2016.

[5] H. Shen, L. Jian, and J. Miao, "Application Review of Network Pharmacology in The Study of Properties Theory of Traditional Chinese Medicine," Chinese Journal of basic Medicine of traditional Chinese Medicine, vol. 25, no. 1, pp. 127-130, 2019.

[6] S. Ying, L. Feng, and L. Yan, "Systems pharmacology (V): research progress in the mechanism analysis of traditional Chinese medicine," Journal of Shenyang Pharmaceutical University, vol. 38, no. 1, pp. 86-94, 2021.

[7] L. Wa, Y. Chunjuan, C. Congcong et al., "Study on the active components and pharmacological effects of Sanguisorba officinalis L. based on network pharmacology," World Science and 
Technology-Modernization of traditional Chinese Medicine, vol. 21, no. 7, pp. 1336-1345, 2019.

[8] L. Jia, S. Qian, Z. Yamei, and H. Lin, "Clinical research progress of non-pharmacological treatment of Alzheimer's disease in Chinese and Western medicine," Modern Journal of Integrated traditional Chinese and Western Medicine, vol. 29, no. 12, pp. 1351-1355, 2020.

[9] G. Yan, S. Wei, and C. Guohua, "Exploration on Medication Rules in Treatment for Alzheimer's Disease with Chinese Materia Medica Based on Data Mining," Journal of Hunan University of Chinese Medicine, vol. 40, no. 12, pp. 15151520, 2020.

[10] G. Jian, Q. Jiayi, W. Yexing, Q. Kuo, M. Yue, and D. Haiying, "Analysis of the dosing pattern of traditional Chinese medicine for Alzheimer's disease," Journal of Qiqihar Medical College, vol. 41, no. 16, pp. 2040-2042, 2020.

[11] Z. Ping, L. Xiaoshuan, W. Hongli, L. Yanyan, and Q. Shenglei, "Progress in Comparative Research on Three Kinds of Calamus," Chinese Journal of Ethnomedicine and Ethnopharmacy, vol. 30, no. 2, pp. 59-62, 2021.

[12] G. Xiuju, H. Hanru, Z. Lijuan, L. Junying, and W. Kangfeng, "The mechanism and clinical application of Renshen (Panax Ginseng) and Shichangpu (Acorus Tatarinowii) in the the prevention and treatment of Alzheimer's disease," Guiding Journal of Traditional Chinese Medicine and Pharmacy, vol. 26, no. 12, pp. 161-163, 2020.

[13] H. Pengyue, L. Guowen, and X. Yan, "Research Advances in mechanism of Rehmannia glutinosa and Acorus calamus in the prevention and treatment of Alzheimer's disease," Journal of Shanghai University of traditional Chinese Medicine, vol. 34, no. 6, pp. 99-104, 2020.

[14] G. Ningxin, Protective Effect and Mechanism of Volatile Oil from Acorus Tatarinowii on Neuronal Injury in AD Model Mice, Guangdong Pharmaceutical University, 2018.

[15] D. Haiying, T. Yaxin, L. Shan, W. Tinghua, X. Yangyanbin, and D. Haiying, "Establishment and Identification of APP/ PS1+SCN2B-/- Transgenic Mouse Model," Journal of Kunming Medical University, vol. 40, no. 12, pp. 7-12, 19.

[16] W. Jing and J. Dong, "Study on clinical effect and partial mechanism of Medullary brain therapy combined with acupuncture on the treatment of senile dementia," Chinese Journal of traditional Chinese Medicine, pp. 1-9, 2021, http://kns.cnki.net/ kcms/detail/21.1546.R.20210205.1504.004.html.

[17] T. Seki, M. Kanagawa, K. Kobayashi et al., "Galectin 3binding protein suppresses amyloid- $\beta$ production by modulating $\beta$-cleavage of amyloid precursor protein," Journal of Biological Chemistry, vol. 295, no. 11, pp. 3678-3691, 2020.

[18] W. Xinhua, "Effects of soybean isoflavones Active-extracts on Activities of ChAT and Caspase-3 in AD Rats with Alzheimer's disease," Journal of Liaoning University of traditional Chinese Medicine., vol. 15, no. 2, pp. 55-56, 2013.

[19] J. Fu, F. Bao, M. Gu et al., "Design, synthesis and evaluation of quinolinone derivatives containing dithiocarbamate moiety as multifunctional AChE inhibitors for the treatment of Alzheimer's disease," Journal of Enzyme Inhibition and Medicinal Chemistry, vol. 35, no. 1, pp. 118-128, 2020.

[20] A. V. McCormick, J. M. Wheeler, C. R. Guthrie, N. F. Liachko, and B. C. Kraemer, "Dopamine D2 receptor antagonism suppresses tau aggregation and neurotoxicity," Biological Psychiatry, vol. 73, no. 5, pp. 464-471, 2013.
[21] L. Ling, G. Liang, Y. Lina, and Z. Gaoli, "Safflower glycosides based on improve spatial memory in Alzheimer's disease rats," Chinese Journal of Gerontology, vol. 41, no. 1, pp. 153-157, 2021.

[22] F. Wencheng, L. Jianping, and L. Changhong, "Roles of PI3K/ AKT Signaling Pathway in Neurodegenerative Diseases," Letters in Biotechnology, vol. 28, no. 6, pp. 853-859, 2017.

[23] H. Ksiezak-Reding, H. K. Pyo, B. Feinstein, and G. M. Pasinetti, "Akt/PKB kinase phosphorylates separately Thr212 and Ser214 of tau protein in vitro," Biochimica et Biophysica Acta, vol. 1639, no. 3, pp. 159-168, 2003.

[24] C. W. Lee, K. F. Lau, C. C. Miller, and P. C. Shaw, "Glycogen synthase kinase- $3 \beta$-mediated tau phosphorylation in cultured cell lines," Neuroreport, vol. 14, no. 2, pp. 257-260, 2003.

[25] Z. Jun and L. Furu, "Progress in the study of the p38 MAPK signaling pathway in relation to Alzheimer's disease," Electronic Journal of Clinical Medical Literature, vol. 5, no. 20, pp. 197-198, 2018.

[26] Z. Chunxia, G. Junhe, C. Yunbo et al., "Effects of active components of Acorus tatarinowii on APP and synaptic ultrastructure in double transgenic mice," New drugs of Traditional Chinese Drug Research and Clinical Pharmacology, vol. 25, no. 1, pp. 18-23, 2014.

[27] W. Xiaoli, D. Miaoxian, X. Tianjiao, L. Chengchong, S. Jikai, and L. Xiaoming, "Protective effects of $\beta$-asarone on primary rat hippcampal neurons against hypoxia/hypoglycemia and reperfusion injury," Chinese Journal of Pathophysiology, vol. 30, no. 5, pp. 928-932, 2014.

[28] N. Kang, Effects and possible mechanism of $\beta$-Asarone on cognitive impairment in ADDITION model rats, Hefei University of Technology, 2020.

[29] M. Deng, L. Huang, B. Ning et al., “ $\beta$-asarone improves learning and memory and reduces acetyl cholinesterase and betaamyloid 42 levels in APP/PS1 transgenic mice by regulating Beclin-1-dependent autophagy," Brain Research, vol. 1652, pp. 188-194, 2016.

[30] S. B. Mesole, O. O. Alfred, U. A. Yusuf, L. Lukubi, and D. Ndhlovu, "Apoptotic inducement of neuronal cells by aluminium chloride and the neuroprotective effect of eugenol in Wistar rats," Oxidative Medicine and Cellular Longevity, vol. 2020, Article ID 8425643, 7 pages, 2020.

[31] V. Deepak, A. Kasonga, M. C. Kruger, and M. Coetzee, "Inhibitory effects of eugenol on RANKL-induced osteoclast formation via attenuation of NF- $\kappa \mathrm{B}$ and MAPK pathways," Connective Tissue Research, vol. 56, no. 3, pp. 195-203, 2015.

[32] H. Liu, X. Lai, Y. Xu et al., “ $\alpha$-Asarone attenuates cognitive deficit in a pilocarpine-induced status epilepticus rat model via a decrease in the nuclear factor- $\kappa \mathrm{B}$ activation and reduction in microglia neuroinflammation," Frontiers in Neurology, vol. 8, p. 661, 2017.

[33] J. W. Shin, Y. J. Cheong, Y. M. Koo et al., " $\alpha$-Asarone ameliorates memory deficit in lipopolysaccharide-treated mice via suppression of pro-inflammatory cytokines and microglial activation," Biomolecules \& Therapeutics, vol. 22, no. 1, pp. 17-26, 2014.

[34] M. Xinxin, Q. Qiankun, T. Yuan, Z. Huan, and G. Liyang, "Effects of paroxetine on Effects of Paroxetine on Alzheimer's Disease Combined with Depression on Serum NE and 5-HT Expression," Progress in Modern Biomedicine, vol. 20, no. 21, article 10.4062/biomolther.2013.102, pp. 4080-4083, 2020. 
[35] M. M. Said and M. M. Rabo, "Neuroprotective effects of eugenol against aluminiuminduced toxicity in the rat brain," Arhiv za Higijenu Rada i Toksikologiju, vol. 68, no. 1, pp. 27-37, 2017.

[36] H. Liping, N. Baile, N. Wang, Z. Qinxin, and M. Deng, "Effects of $\beta$-asarone combined with levodopa on molecular chaperone-mediated autophagy in 6-OHDA-induced Parkinson's model rats," Journal of Chinese Medicinal Materials, vol. 43, no. 7, pp. 1720-1724, 2020.

[37] S. Nakajima, P. Gerretsen, H. Takeuchi et al., "The potential role of dopamine $\mathrm{D}_{3}$ receptor neurotransmission in cognition," European Neuropsychopharmacology, vol. 23, no. 8, pp. 799-813, 2013.

[38] Z. Xuejiao, J. Hui, T. Susu, and O. Yu, “The role of estrogen receptors in central nervous system diseases," Journal of Pharmaceutical Research, vol. 37, no. 4, pp. 221-225, 2018.

[39] X. Y. Hu, S. Qin, Y. P. Lu, R. Ravid, D. F. Swaab, and J. N. Zhou, "Decreased estrogen receptor-? expression in hippocampal neurons in relation to hyperphosphorylated tau in Alzheimer patients," Acta Neuropathologica, vol. 106, no. 3, pp. 213-220, 2003.

[40] J. Li, Y. He, Y. Zou, and Y. Fang, "Effects of $\beta$-asarone on intracellular calcium concentration in cortical neurons of dementia mice," Chinese Journal of Rehabilitation Medicine, vol. 6, no. 6, pp. 490-491, 2007.

[41] D. Barh, M. E. García-Solano, S. Tiwari et al., "BARHL1 is downregulated in Alzheimer's disease and may regulate cognitive functions through ESR1 and multiple pathways," Genes, vol. 8, no. 10, p. 245, 2017. 